\title{
Supporting \\ Acquisition of Basic Skills
}

\author{
Neville Holmes \\ University of Tasmania
}

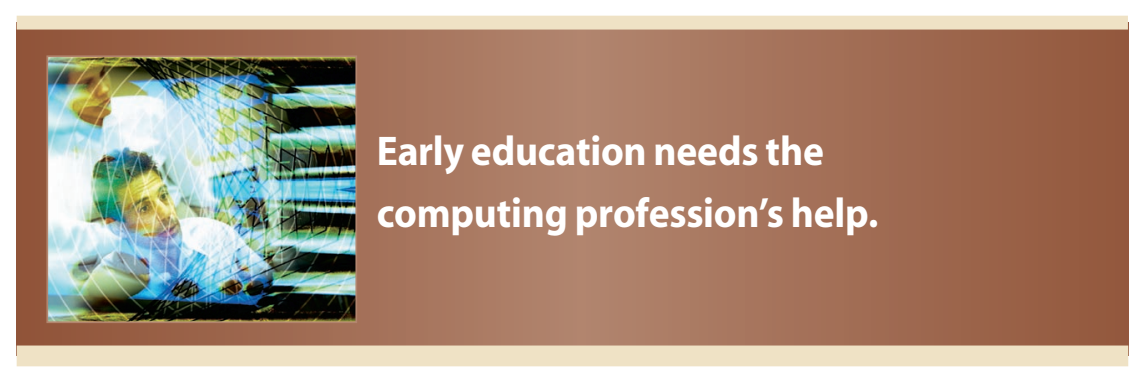

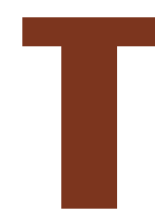

like this:

he storyline of my March 2007 essay, "Digital Technology and the Skills Shortage" (The Profession, pp. 100, 98-99), went

- Skills you don't have to think about using are basic.

- Basic skills underpin advanced skills and accumulate through drill and practice.

- The younger the learner, the easier it is to acquire basic skills.

- Traditional classroom drill is largely boring and unsuccessful for many.

- Computer-delivered individual drill and practice could be hugely successful.

Convinced of the benefits of computer-delivered drill, and persuaded of the urgent need for those benefits to be gained, I discussed the issues with teachers and computing professionals at conferences and privately. It became clear that the benefits and ways to gain them are not as obvious as I had thought.

\section{FOR TEACHERS}

Teachers and parents have two educational roles with respect to their charges: instructor and mentor. Instructors induce aptitudes: abstract skills and knowledge. Mentors foster attitudes: social skills and graces.

In my experience of school, starting 70 years or so ago, quite regimented instruction predominated in the earlier stages, with the aim of developing basic skills by repetition. My strong impression is that the pendulum has now swung to the opposite extreme, at least in the countries most familiar to me.

This is tragic, as both abstract and social skills are left greatly underdeveloped in many young people when they leave school. Because the development of attitudes depends on the possession of abstract skills such as oralcy, numeracy, and literacy (both grammar and vocabulary), teachers and parents are greatly restricted in fulfilling the mentor role. In failing to influence their attitudes, they let children's minds become dominated by their senses and perceptions rather than by their social imagination and sensitivity. This has led to young people nowadays often being referred to as the "me first" generation.

The pity of this is that computers can deliver traditional drill automatically and vastly more effectively than teachers. Such automatic drill and practice can be designed to adapt to the capabilities, moods, and interests of individual learners and to collect data on learners' progress that mentors can use in designing social activities and selecting subsequent areas for drill. This would let individual learners develop their basic skills in different ways and at different rates, and it would let teachers detect and act to remedy defects and imbalance as they become evident.

The implications are profound. The traditional class structure becomes irrelevant if not obstructive, and the traditional periodic tests and examinations become pointless. The teacher's role becomes communal and participative rather than individual and supervisory.

\section{AUTOMATIC DRILL}

The rich possibilities of automatic drill might be better appreciated by considering a specific example. Suppose the objective is to create a drill package that supports learners in building a vocabulary about pets-say, dogs (en.wikipedia.org/ wiki/Dogs for background).

The first goal is acquiring the ability to recognize and name dogs. The first stage would be distinguishing between dogs and other canids, such as wolves, foxes, jackals, coyotes, and hyenas. The second stage would be distinguishing among various types and breeds of domestic dogs.

A drill set consists of items to be learned. In our example, the items are canids in the first stage and domestic dogs in the second. The main components of an item are the name to be learned and several depictions of what it names.

Continued on page 102 
A drill session is a sequence of settings for groups of items chosen from the set to best promote learning. A setting starts by displaying either a randomly chosen depiction from each item of the group, or of the name from each item-sometimes one, sometimes the other. A setting continues by presenting, one at a time in random sequence, the other components of a subset of the items of the group. If the names are static, a depiction is randomly chosen for dynamic display from each item.

Each dynamic component appears only for a short time, and the learner selects by voice or touch the corresponding static component. If the objective is to train short-term memory as well as recognition, the duration of presentation can be made shorter than that allowed for the learner's reaction.

Each setting ends with a small report on performance, including a short corrective message for any item in error. Then the learner can choose brief tutoring for any items in error, more detailed tutoring for any item of the set, the option of following relevant Web links provided for further information, or finishing the session. Such tutoring and links are secondary components of each item.

At the beginning of a drill session, the software will use details of each learner's performance so far, to allow reinforcement of items introduced in recent sessions, before introducing any new items. Items will be classified as the learner progresses into categories such as pending, just introduced, recently learned, and solidly learned. This classification guides the learning process, partly by presenting betterlearned items less frequently and perhaps more briefly.

The learner's use of a drill package will go through phases. Before the learner starts, all items will be classed as pending, with a preliminary phase needed to determine what the learner already knows, how new items will be introduced, what reaction time is natural, and coverage of basic tutoring, if necessary. Then new items will be gradually introduced, guided by item interdependencies specified in the item data and by the learner's performance. There should be more items and depth than all but very quick learners can cover in the time available, but if ever all items have been solidly learned, the package can be used repeatedly in a final refresh phase.

\section{Automatic drill challenges learners individually, much as do videogames.}

\section{OBSERVATIONS}

Drill packages of this kind can be made enormously adaptable because there are so many parameters that determine just how the drill will proceed. While some static parameters are inherent in the item data-specifying precedence, subsequence, and other relations between items - the dynamic parameters specific to the individual learner let the drill be optimized for different people of different ages at different times, and everyone's learning sequence and attainment become different in detail.

The point of learning vocabulary is to enrich other activities, especially social activities. At school, this means the teacher must devise group activities based on the acquired vocabulary and aimed at the interactive extension of knowledge in that area and at the development of basic social skills. The parents also can use a drill package that their child uses so that they can interact to reinforce and motivate the learning.

Automatic drill aims to improve and extend basic skills. Successful learning is therefore shown as much by speed of response as by correctness. Speed is built up by repetition, the latter part of drill and practice. Repetition is generally held to be boring by teachers, and it certainly was, when done as a class. But automatic drill challenges learners individually, much as do videogames.

Drill packages can be adapted for the very young by using digital speech production and recognition technology. In the preceding example, if the dynamic component of a setting were the name, it could be spoken instead of, or as well as, written, and the response could be by touch selection. If the static components were names, the child could select the name for a depiction by speaking it, and tutoring could be given on the spot to improve pronunciation. By using headsets with ear microphones, this would even be practical in a noisy schoolroom.

Drill for numeracy could have much the same structure as that of the example above and, because the organization would be much more regular, subitising and arithmetic could be effectively taught, particularly with the use of speech recognition. Sentence completion for grammar is straightforward, but much more variety is needed. Spelling drill for depictable words could be done through a keyboard. Learning to spell by speech recognition might, however, need more distinctive names for the alphabet's letters to be really effective, such as those suggested in my March 2007 essay. Grammatical words and homophones also present a challenge.

\section{DRILL AND THE PROFESSION}

In the last year or so, many drill packages have become available under various guises and running on a variety of equipment. However, these are mostly designed to be marketed, not to educate.

We face the grave danger that automatic drill will go the way of radio, television, and, to a lesser degree, the Web. All were agreed to have tremendous educational potential when introduced, yet all have been turned or are turning into tools for marketing. Even videogames and social websites have been infiltrated by marketing ploys such as product placement.

If we let marketing predominate, automatic drill's potential will be lost. The most marketable drill packages will come and go, suffering from the 
customary feature bloat and interface cancer. Programmers will develop them independently of teachers and they will be used for distraction rather than improvement.

Marketing activity will always be present and will always loom large. We must ensure that it doesn't predominate in schools, however, and then it needn't predominate in the home. This can only be achieved if the teaching and computing professions cooperate. It cannot be done by confronting the commercial world.

The key here is to realize that the essential component of automatic drill is the drill data, not the programs that use that data. The two professions must collaborate to develop and control a standard for drill data independent of any particular equipment using that data. The standard would need to specify the representation of the static data for a package (the data before it is used) and the dynamic data (the data generated about each learner). A standard for compilation of extracted performance data also might be worthwhile.

Three classes of software would be needed to exploit the standard: compilation software for putting drill packages together, delivery software for running the drill, and evaluation software for teachers and parents to use and monitor the drill.

Early versions of such software would be written by or for researchers during the standard's development. Success in its early use would divert at least some of the commercial software world's attention to providing versions with improvements or adaptations to use different equipment, ranging from video consoles to mobile phones. The danger here is that the commercial world will fiddle with the standard to gain what it sees as a marketing advantage. The influence of the two professions will be needed to prevent this from happening.

The drill data standard must allow a wide range of drill types and styles. Teachers need the ability to put together very small packages for special day-to-day educational purposes that run on laptops, and large educational enterprises such as Encyclopedia Britannica and Wikipedia must be able to put together and extend huge packages for the public to use from the Web.

Automatic drill is potentially useful to all segments of society. Because the potential is greatest for preschool and elementary education, however, priority must be given to exploiting speech recognition technology once the most basic standard has been accepted.

$\mathbf{T}$ he significance of automatic drill to the computing profession is profound. The outline I have provided merely suggests the technology's huge potential. The effect of greatly increasing the basic skills of primary school graduates would be amplified in turn at the secondary and tertiary stages, both of which could also use automatic drill to amplify graduate skills.

The significance is not just localit's international, in two ways. First, automatic drill has even greater potential in underdeveloped countries than in developed ones. If a special-purpose driller were developed, it could be much cheaper and far more beneficial than the $\$ 100$ laptop. Second, automatic drill could make all primary school graduates bilingual and thus able to communicate with native speakers of their second language over the Internet, and indeed it could greatly help monolingual adults become bilingual.

The need is urgent. The computing and teaching professions have two responsibilities: to get that need widely and politically accepted and to begin working together to fulfill that need.

Neville Holmes is an honorary research associate at the University of Tasmania's School of Computing and Information Systems. Contact him at neville.holmes@utas.edu.au.

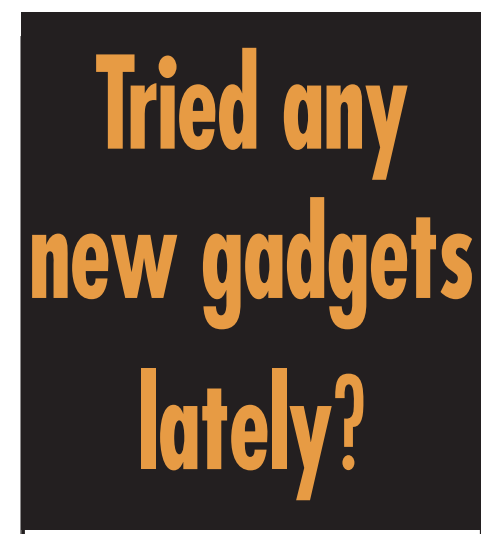

Any products your peers should know about? Write a review

for IEEE Pervasive

Computing, and tell us why you were

impressed. Our New

Products department

features reviews of the latest components,

devices, tools, and other ubiquitous computing gadgets on the market.

Send your reviews and recommendations to pvcproducts@ computer.org today!

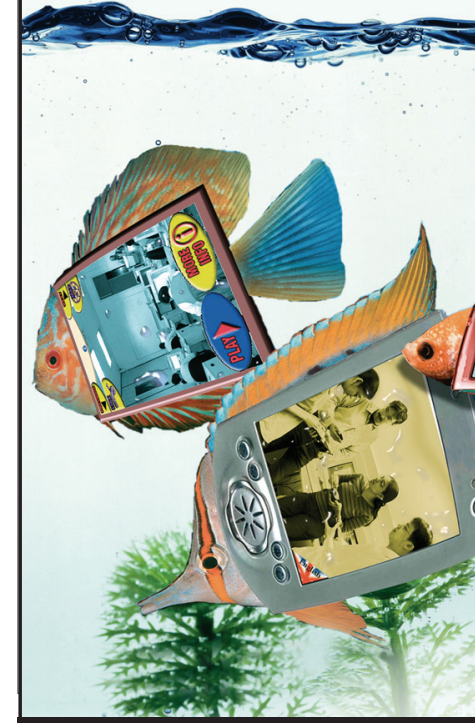

www.computer.org/pervasive 